\title{
THE REGULARITY OF A GENUS OF POSITIVE TERNARY QUADRATIC FORMS*
}

\author{
BY \\ BURTON W. JONES $†$
}

1. Introduction. L. E. Dickson $\ddagger$ has defined a positive ternary quadratic form $f$ to be regular when the integers not represented by the form $f$ coincide with certain arithmetic progressions.

The methods previously used for proving a form regular have been chiefly modifications $\S$ of a method used by Dirichlet $\|$ for the form $x^{2}+y^{2}+z^{2}$ together with certain elementary methods by which results for one form have been derived as corollaries from those of another. Now Dirichlet's method or modifications of it may be applied only when there is but one form in the genus or when it can otherwise be proved that all the integers represented by the forms of a genus are represented by one form. Heretofore it has been necessary to carry out a Dirichlet type of proof for each separate form or very restricted set of forms unless the proof could be referred back by elementary means to previous results for other forms, that is, with the exception of two cases when elliptic functions have been used.

In this paper it is proved that the integers represented by all of the forms of a genus $\uparrow$ coincide with the positive integers in certain arithmetic progressions, i.e., a form $f$ of genus $G$ is regular if and only if $f$ represents all the integers represented by every form of $G$. Using this result in the case of any form $f$, in order to prove $f$ regular, it is necessary merely to show that there is only one class in the genus of $f$ or that every integer represented by a form of the genus is represented by $f$. Similarly this establishes a new criterion for irregularity. Furthermore the form of these progressions in terms of the generic invariants is given by the author in his previous paper.

Examples are given at the end of this paper. Throughout this paper $f$

* Presented to the Society, December 30, 1930; received by the editors in July, 1930.

$\dagger$ National Research Fellow.

$\ddagger$ Annals of Mathematics, (2), vol. 28 (1927), pp. 333-341.

$\S$ See Annals of Mathematics, ibid.; Bulletin of the American Mathematical Society, vol. 33 (1927), p. 63.

|| Journal für Mathematik, vol. 40 (1850), pp. 228-32.

If For the definition of genus see H. J. S. Smith, Collected Papers, vol. 1, pp. 455-509, and A new definition of genus for ternary quadratic forms by the author, in the present number of these Transactions, referred to in this article as "the previous paper." L. E. Dickson in his definition of genus (Studies in the Theory of Numbers, p. 52) omits certain of Smith's generic characters because of their redundancy. It is immaterial in this paper whether Dickson's or Smith's definition is used. 
denotes a primitive positive ternary quadratic form. In the opinion of the author, this discussion could be easily modified to include indefinite forms, also, but he has refrained from doing this in order to avoid possible duplication with a paper on indefinite forms by Mr. Arnold Ross soon to appear in the Proceedings of the National Academy and Sciences.

2. Lemmas. We shall first prove some elementary lemmas.

LEMMA 1. If $a_{1}$ is any integer primitively represented by a primitive form $f=(a, b, c, 2 r, 2 s, 2 t)$, i.e., $f=a x^{2}+b y^{2}+c z^{2}+2 r y z+2 s x z+2 t x y, f$ is equivalent to a form with leading coefficient $a_{1}$.

This has been proved elsewhere but the proof is so simple that it is given here for completeness.

Suppose a primitive solution of $f=a_{1}$ is $\left(x_{1}, y_{1}, z_{1}\right)$. Let $\left(x_{1}, y_{1}\right)=g$, the g.c.d. of $x_{1}$ and $y_{1}$, and choose integers $u$ and $v$ such that $x_{1} v-y_{1} u=g$. Then $g$ is prime to $z_{1}$ since the solution is primitive and there thus exist a $k$ and an $n$ such that $g k-z_{1} n=1$. Then the transformation

$$
x=x_{1} X+u Y+x_{1} n Z / g, \quad y=y_{1} X+v Y+y_{1} n Z / g, z=z_{1} X+k Z
$$

is of determinant 1 , has integral coefficients, and takes $f$ into a form with leading coefficient $a_{1}$.

Lemma 2. For any prime $q$ and any positive integer $k$, the replacement of $z$ by $z+\tau_{1} y$ and then $y$ by $y+\tau_{2} z$ in $f=\left(a_{1}, b, c, 2 r, 2 s, 2 t\right)$ yields, for a proper choice of $\tau_{1}$ and $\tau_{2}\left(\bmod q^{k}\right)$, a form

with $s^{\prime} \equiv t^{\prime}\left(\bmod q^{k}\right)$.

$$
f^{\prime}=\left(a_{1}, b^{\prime}, c^{\prime}, 2 r^{\prime}, 2 s^{\prime}, 2 t^{\prime}\right)
$$

If we subject $f$ to such a transformation, we see the coefficient of $x^{2}$ remains unaltered, $t^{\prime}=t+s \tau_{1}$ and $s^{\prime}=t \tau_{2}+s\left(1+\tau_{1} \tau_{2}\right)=\tau_{2}\left(t+s \tau_{1}\right)+s$. If $s$ is prime to $q$, take $\tau_{2} \equiv 0 \quad\left(\bmod q^{k}\right)$ and have $s^{\prime} \equiv s \quad\left(\bmod q^{k}\right)$. Then $\tau_{1}$ may be chosen $\left(\bmod q^{k}\right)$ so that $t^{\prime}=t+s \tau_{1} \equiv s \quad\left(\bmod q^{k}\right)$. If $t$ but not $s$ is prime to $q$, proceed similarly. If $s \equiv 0 \equiv t \quad(\bmod q)$ and $k>1$, replace $s, t, s^{\prime}$ and $t^{\prime}$ above by $s / q$, $t / q, s^{\prime} / q, t^{\prime} / q$ respectively, and prove in precisely the same manner that $\tau_{1}$ and $\tau_{2}$ may be so chosen that $s^{\prime} / q \equiv t^{\prime} / q \quad\left(\bmod q^{k-1}\right)$ unless $s \equiv t \equiv 0 \quad\left(\bmod q^{2}\right)$ and $k>2$. This process may be continued until the proof is complete.

Consider a form $f=\left(a_{1}, b, c, 2 r, 2 s, 2 t\right)$. Transform it first by

$$
x=x^{\prime}+\tau y+\sigma z, y=y, z=z,
$$

and have

$$
\begin{aligned}
f^{\prime}= & a_{1} x^{\prime 2}+\left(a_{1} \tau^{2}+2 t \tau+b\right) y^{2}+\left(a_{1} \sigma^{2}+2 s \sigma+c\right) z^{2}+2\left(r+a_{1} \tau \sigma+t \sigma+s \tau\right) y z \\
& +2\left(a_{1} \sigma+s\right) x z+2\left(a_{1} \tau+t\right) x y \\
= & a_{1} x^{\prime 2}+b^{\prime} y^{2}+c^{\prime} z^{2}+2 r^{\prime} y z+2 s^{\prime} x z+2 t^{\prime} x y .
\end{aligned}
$$


Let $\lambda=\left(s^{\prime}, t^{\prime}\right)$ and transform $f^{\prime}$ by

$$
x^{\prime}=x^{\prime}, y=\alpha y^{\prime}+\beta z^{\prime}, z=\gamma y^{\prime}+\delta z^{\prime} ;
$$

where

$$
\alpha=-s^{\prime} / \lambda, \gamma=t^{\prime} / \lambda,
$$

and $\beta$ and $\delta$ are chosen so that

$$
\alpha \delta-\beta \gamma=1 .
$$

This yields

$f^{\prime \prime}=a_{1} x^{\prime 2}+b^{\prime \prime} y^{\prime 2}+c^{\prime \prime} z^{\prime 2}+2 r^{\prime \prime} y^{\prime} z^{\prime}+2 s^{\prime \prime} x^{\prime} z^{\prime}$ where $b^{\prime \prime}=\alpha^{2} b^{\prime}+\gamma^{2} c^{\prime}+2 r^{\prime} \alpha \gamma$.

Let $\Omega$ denote the g.c.d. of the literal coefficients of the form adjoint to $f$, i.e. the g.c.d. of $b c-r^{2}, a_{1} c-s^{2}, a_{1} b-t^{2}, s t-a_{1} r, r t-b s, r s-c t$. Let $q$ be 2 or any odd prime factor of the Hessian $H$ of a primitive form $f=\left(a_{1}, b, c, 2 r, 2 s, 2 t\right)$ and $\epsilon$ the highest power of $q$ occurring as a factor of $H$. We prove

Lemma 3. If $a_{1} \neq 00(\bmod q)$ and $\Omega \equiv 0\left(\bmod q^{\omega}\right)$ but $\Omega \not \equiv 0\left(\bmod q^{\omega+1}\right)$, where $\omega$ is a positive integer or zero, it is true that $\tau_{1}$ and $\tau_{2}$ in Lemma 2 and $\tau$ and $\sigma$ in (1) may be so chosen modulo $q$ or a power of $q$ that (2) yields a form $f^{\prime \prime}$ with $b^{\prime \prime} \equiv r^{\prime \prime} \equiv 0 \quad\left(\bmod q^{\omega}\right)$ but $b^{\prime \prime} \not \equiv 0 \quad\left(\bmod \pi q^{\omega+1}\right)$, where $\pi=2$ or 1 according as $q$ is even or odd.

First note that $\Omega$, being an invariant under transformations (1) and (2), is a divisor of $a_{1} b^{\prime \prime}$ and $a_{1} r^{\prime \prime}$, thus showing $b^{\prime \prime} \equiv r^{\prime \prime} \equiv 0 \quad\left(\bmod q^{\omega}\right)$. From Lemma 2 we may consider the following congruence to hold: $t \equiv s\left(\bmod q^{\epsilon+2}\right)$. Note that $2 \omega \leqq \epsilon$.

Now if $a_{1} b \neq t^{2} \quad\left(\bmod \pi q^{\omega+1}\right)$, choose $\tau$ so that $a_{1} \tau+t=t^{\prime} \equiv 0 \quad\left(\bmod \pi q^{\omega+1}\right)$ and $\sigma$ so that $a_{1} \sigma+s=s^{\prime} \not \equiv 0(\bmod q)$. Then $\gamma \equiv 0 \quad\left(\bmod \pi q^{\omega+1}\right)$ and $b^{\prime \prime}$ $\equiv \alpha^{2}\left(a_{1} \tau^{2}+2 t \tau+b\right) \equiv \alpha^{2}(t \tau+b) \not \equiv 0\left(\bmod \pi q^{\omega+1}\right)$ since $a_{1}(t \tau+b) \equiv-t^{2}+a_{1} b \neq 0$ $\left(\bmod \pi q^{\omega+1}\right)$. If $a_{1} c \neq t^{2} \quad\left(\bmod \pi q^{\omega+1}\right)$ the proof is similar.

If $a_{1} b \equiv a_{1} c \equiv t^{2} \quad\left(\bmod \pi q^{\omega+1}\right)$ and $a_{1} r \not \equiv t^{2} \quad\left(\bmod q^{\omega+1}\right)$, choose $\tau$ and $\sigma$ so $t^{\prime} \equiv s^{\prime} \equiv 0 \quad\left(\bmod \pi q^{\omega+1}\right)$ and $\alpha \gamma \neq \equiv 0 \quad(\bmod q)$. Then $b^{\prime \prime} \equiv \alpha^{2}(t \tau+b)+\gamma^{2}(t \sigma+c)$ $-2(r+s \tau) \alpha \gamma \equiv-2(r+t \tau) \alpha \gamma \neq 0 \quad\left(\bmod \pi q^{\omega+1}\right)$.

Finally $a_{1} b \equiv a_{1} c \equiv a_{1} r \equiv t^{2} \quad\left(\bmod q^{\omega+1}\right)$ implies $\Omega \equiv 0 \quad\left(\bmod q^{\omega+1}\right)$ which is contrary to hypothesis.

LEMMA 4. If $a_{1} \equiv 0 \quad\left(\bmod q^{\omega_{1}}\right)$ where $\omega_{1} \geqq 1, \Omega \neq 0(\bmod q)$ and $q$ is 2 or an odd prime factor of $H$, then, for the proper choice in (1) of $\tau$ and $\sigma$ and $\tau_{1}$ and $\tau_{2}$ in Lemma 2 modulo $q$ or a power of $q$, (2) yields a form $f^{\prime \prime}$ with $b^{\prime \prime} \not \equiv 0$ $\left(\bmod \pi q^{\omega_{1}+1}\right)$ where $\pi=2$ or 1 according as $q$ is even or odd and $a_{1} \neq 0\left(\bmod q^{\omega_{1}+1}\right)$.

From Lemma 2 we need consider only $s \equiv t \quad\left(\bmod q^{2 \omega_{1}+3}\right)$. Then suppose 
$s \equiv t \equiv 0 \quad\left(\bmod q^{\omega_{2}}\right)$ where $\omega_{2}$ is the greatest integer for which the congruence holds.

First: $\omega_{2} \geqq \omega_{1}>0$. If $b \not \equiv 0(\bmod q)$ choose $\tau$ and $\sigma$ so that $\alpha \neq 0 \equiv \gamma$ $(\bmod q)$. Then $b^{\prime \prime} \equiv \alpha^{2} b \neq 0 \quad(\bmod q)$. Proceed similarly if $c \neq \equiv 0 \quad(\bmod q)$. If $b \equiv c \equiv 0 \quad(\bmod q)$ we know $r \neq 0 \quad(\bmod q)$, since $f$ is primitive, and, choosing $\tau$ and $\sigma$ that so $\alpha \gamma \neq \equiv(\bmod q)$ we have $b^{\prime \prime} \equiv 2 r \alpha \gamma \neq \equiv 0(\bmod q)$ unless $q=2$. If $b+c \equiv 0 \quad(\bmod 4)$, choose $\sigma$ and $\tau$ divisible by 4 , and so that $\alpha \gamma$ is odd, and have $b^{\prime \prime} \equiv b+c+2 r \equiv 2 \quad(\bmod 4)$. If $b+c \equiv 2 \quad(\bmod 4)$ and $b$ and $c$ are even, we complete the proof for $\omega_{2} \geqq \omega_{1}>0$ as follows.

(1) If $t \equiv 0 \equiv b+2 \quad(\bmod 4)$ take $\tau$ so that $\gamma \equiv a_{1} \tau+t \equiv 0 \quad(\bmod 4)$ and $\sigma$ so that $\alpha$ is odd, and see $b^{\prime \prime} \equiv t \tau+b \equiv 2 \quad(\bmod 4)$. Make $\gamma$ odd and $\alpha \equiv 0$ $(\bmod 4)$ if $c \equiv 2(\bmod 4)$.

(2) If $t \equiv 2 \equiv a_{1} \quad(\bmod 4)$ and $b \equiv 0 \quad(\bmod 4)$, take $\tau$ odd and $\sigma$ even, have $\alpha$ odd, $\gamma$ even and $b^{\prime \prime} \equiv a_{1} \equiv 2 \quad(\bmod 4)$. Take $\tau$ even and $\sigma$ odd if $c \equiv 0$ $(\bmod 4)$.

Second: $\omega_{2}=0$. Note $\alpha \gamma \neq \equiv 0 \quad(\bmod q)$ and let $\omega_{3}$ be the greatest integer for which $b+c-2 r \equiv 0 \quad\left(\bmod q^{\omega_{3}}\right)$. If $\omega_{3} \leqq \omega_{1}$, take $\tau \equiv \sigma \equiv 0 \quad\left(\bmod q^{\omega_{3}+1}\right)$ so that $\alpha \equiv-\gamma \quad\left(\bmod q^{\omega_{3}+1}\right)$, and have $b^{\prime \prime} \equiv \alpha^{2}(b+c-2 r) \not \equiv 0 \quad\left(\bmod q^{\omega_{3}+1}\right)$. If $\omega_{1}<\omega_{3}$, take $\sigma \equiv 0\left(\bmod q^{\omega_{1}+1}\right)$, and have $b^{\prime \prime} \equiv \alpha^{2}\left(a_{1} \tau^{2}+2 t \tau+b\right)+\gamma^{2} c+2(r$ $+t \tau) \alpha \gamma \quad\left(\bmod q^{\omega_{1}+1}\right)$. Noting $\gamma \lambda=t+a_{1} \tau$ and $\alpha \lambda \equiv-t \quad\left(\bmod q^{\omega_{1}+1}\right)$, where $\lambda$ is prime to $q$, we have $b^{\prime \prime} \lambda^{2} \equiv t \tau a_{1}\{-t \tau+2(c-r)\} \quad\left(\bmod q^{\omega_{1}+1}\right)$ and $\tau$ may be chosen prime to $q$ so that $b^{\prime \prime} \not \equiv 0\left(\bmod q^{\omega_{1}+1}\right)$.

Third: $\omega_{1}>\omega_{2}>0$. Note $\alpha \gamma \neq 0 \quad(\bmod q)$. If $\omega_{3} \leqq \omega_{1}-\omega_{2}+\pi$ take $\tau \equiv \sigma \equiv 0$ $\left(\bmod q^{\omega_{1}-\omega_{2}+3}\right)$, so that $\alpha \equiv-\gamma \quad\left(\bmod q^{\omega_{1}-\omega_{2}+3}\right)$, and have $b^{\prime \prime} \equiv \alpha^{2}(b+c-2 r)$ $\not \equiv 0 \quad\left(\bmod q^{\omega_{1}-\omega_{2}+\pi+1}\right)$, i.e. $b^{\prime \prime} \not \equiv 0 \quad\left(\bmod \pi q^{\omega_{1}+1}\right)$. If $\omega_{3}>\omega_{1}-\omega_{2}+\pi$, note that $b \neq r \neq \equiv c \quad(\bmod q)$, since $b+c-2 r \equiv 0 \quad(\bmod q)$ and $b \equiv r \quad(\bmod q)$ would imply $c-r \equiv b c-r^{2} \equiv \Omega \equiv 0 \quad(\bmod q)$ contrary to hypothesis. Now take $\sigma \equiv 0$ $\left(\bmod q^{\omega_{1}+\omega_{2}+3}\right)$ and have, as in the paragraph above, $b^{\prime \prime} \lambda^{2} / q^{2 \omega_{2}} \equiv t a_{1} \tau(-t \tau$ $+2\{c-r\}) / q^{2 \omega_{2}}+a_{1}^{2} \tau^{2} c / q^{2 \omega_{2}} \quad\left(\bmod q^{\omega_{1}-\omega_{2}+\pi+1}\right)$.

Now if $q=p$ an odd prime, the first member on the right of the last congruence is $\equiv 2 \tau t a_{1}(c-r) / p^{2 \omega_{2}} \quad\left(\bmod p^{\omega_{1}-\omega_{2}+1}\right)$, and the second $\equiv 0 \quad(\bmod$ $\left.p^{\omega_{1}-\omega_{2}+1}\right)$ since $2 \omega_{1}-2 \omega_{2} \geqq \omega_{1}-\omega_{2}+1$. Thus, taking $\tau \neq \equiv 0 \quad(\bmod p)$ we have $b^{\prime \prime} \not \equiv 0\left(\bmod p^{\omega_{1}-\omega_{2}+1}\right)$, i.e., $b^{\prime \prime} \not \equiv 0 \quad\left(\bmod p^{\omega_{1}+1}\right)$.

If $q=2$ we have $c-r \equiv 1(\bmod 2)$, and thus $b^{\prime \prime} \equiv A \cdot 2^{\omega_{1}} \tau^{2}+B \tau \cdot 2^{\omega_{1}-\omega_{2}+1}$ $+C \tau^{2} \cdot 2^{2 \omega_{1}-2 \omega_{2}}\left(\bmod 2^{\omega_{1}+3-\omega_{2}}\right)$, where $A B$ is odd and $C \equiv c \quad(\bmod 4)$. Call $C \equiv 2^{\omega_{4}}\left(\bmod 2^{\omega_{4}+1}\right), \omega_{4} \geqq 0$. Then $\tau$ may be chosen so that $b^{\prime \prime} \not \equiv 0(\bmod$ $2^{\left.\omega_{1}+3-\omega_{2}\right)}$ as follows.

(1) If one of $\omega_{1}-\omega_{2}+1, \omega_{1}, 2 \omega_{1}-2 \omega_{2}+\omega_{4}$ is less than the other two, $\tau \equiv 1$ $\left(\bmod 2 \omega_{1}+3-\omega_{2}\right)$. 
(2) If $\omega_{1}-\omega_{2}+1=\omega_{1} \leqq 2 \omega_{1}-2 \omega_{2}+\omega_{4}, \tau$ may be chosen odd so that $\tau A+B$ $\equiv 2(\bmod 4)$ or $\equiv 0 \quad(\bmod 4)$ according as $2 \omega_{1}-2 \omega_{2}+\omega_{4} \geqq \omega_{1}+2$ or not.

(3) If $\omega_{1}-\omega_{2}+1=2 \omega_{1}-2 \omega_{2}+\omega_{4}<\omega_{1}$, we have $\omega_{4}=0, \omega_{1}=1+\omega_{2}$, and choose $\tau$ odd so that $\tau(B+C \tau) \equiv 2$ or $0 \quad(\bmod 4)$ according as $\omega_{1} \geqq \omega_{1}-\omega_{2}+3$ or not. Now $2 \omega_{1}-2 \omega_{2}+\omega_{4}<\omega_{1}-\omega_{2}+1$ is impossible since $\omega_{1}-\omega_{2}+\omega_{4} \geqq 1$. This completes the proof if we note $\omega_{1}+3-\omega_{2} \leqq \omega_{1}+2$.

THEOREM 1. Any form $f$ of Hessian $H$ representing primitively an integer $a_{1}$ prime to $\Omega$ is equivalent to a form $f=a_{1} x^{2}+b y^{2}+c z^{2}+2 r y z+2 s x z$ with $b=\Omega m \beta$, where $m=m_{1} m_{2}, m_{1}$ being prime to $\Omega$ and a divisor of $a_{1}$ and therefore of $H, m_{2}=1$ or $2, \beta$ prime to $2 a_{1} H$, and $c \equiv s \equiv r \equiv 0 \quad(\bmod \Omega)$. ( $\Omega$ is defined just preceding Lemma 3.)

Since the choice of $\tau$ and $\sigma$ in Lemmas 3 and 4 and $\tau_{1}$ and $\tau_{2}$ in Lemma 2 is purely congruential $\bmod q$ or powers of $q$ for each $q$, a choice of $\tau$ and $\sigma$, $\tau_{1}$ and $\tau_{2}$ simultaneously fulfilling the requirements for every $q$ ( 2 or a prime factor of $H$ ) is possible in Lemmas 2, 3, and 4, thus proving that we can make $b=\Omega m \beta$ as in the theorem. By Lemma 3 we have $r \equiv 0 \quad(\bmod \Omega)$. To make $s \equiv 0 \quad(\bmod \Omega)$ after the other conditions of the theorem, except for that on $c$, are satisfied, replace $x$ by $x+\tau z$, note that the coefficients of $x^{2}$ and of the terms in $y$ remain unaltered and that the coefficient of $2 x z$ becomes $a_{1} \tau+s$. $\tau$ may be chosen so that $a_{1} \tau+s \equiv 0 \quad(\bmod \Omega)$. Thus we may consider $s$ in $f$ to be $\equiv 0 \quad(\bmod \Omega)$. Then $a_{1} c-s^{2} \equiv 0 \quad(\bmod \Omega)$ implies $c \equiv 0 \quad(\bmod \Omega)$ and the proof is complete.

3. Consider the forms $f=(a, b, c, 2 r, 2 s, 0)$ and $f^{\prime}=\left(a^{\prime}, b^{\prime}, c^{\prime}, 2 r^{\prime}, 2 s^{\prime}, 0\right)$ of the same Hessian $H$. Let the adjoints of the two forms be $\mathcal{F}$ and $\mathcal{F}^{\prime}$ and the reciprocal forms $F=\mathscr{F} / \Omega$ and $F^{\prime}=\mathscr{F}^{\prime} / \Omega^{\prime}$ respectively. We prove

TheOREM 2. Two forms $f$ and $f^{\prime}$ of Hessian $H$ and having the same properties as $f$ in Theorem 1 are of the same genus if $a \equiv a^{\prime}(\bmod 8 h \mu), b \equiv b^{\prime}, c \equiv c^{\prime}$, $r \equiv r^{\prime}, s \equiv s^{\prime} \quad(\bmod 8 h \Omega)$, where $h$ is the product of the first powers of the odd prime factors of $H$ and $\mu$ is the smallest integer for which a/ $\mu$ is an integer prime to $2 h$.

It has been proved by H. J. S. Smith in his article previously referred to, that the genus of a form depends solely on the quadratic character of the integers represented by $f$ and $F$ with respect to the odd prime factors of the Hessian, the congruences mod 8 satisfied by the odds represented by $f$ and $F$ and, in certain cases, certain so-called "simultaneous characters." Two forms $f$ and $f^{\prime}$ of the same Hessian are obviously of the same character with respect to $8 h$ if the respective literal coefficients are $\equiv(\bmod 8 h)$. Now $a c-s^{2} \equiv a^{\prime} c^{\prime}-s^{\prime 2}$ $(\bmod 8 h \Omega)$ since $a c / \Omega \equiv a^{\prime} c^{\prime} / \Omega(\bmod 8 h)$. The same is true of the other literal coefficients of $\mathcal{F}$ and $\mathcal{F}^{\prime}$. Thus $\Omega^{\prime}=\Omega$, since any factor of $2 h$ dividing 
all the literal coefficients of $\mathcal{F} / \Omega$ divides all the literal coefficients of $\mathcal{F}^{\prime} / \Omega$ and conversely. Thus the literal coefficients of $F$ and $F^{\prime}$ are respectively congruent mod $8 h$. It is easily seen that this implies that the orders and the simultaneous characters of $f$ and $f^{\prime}$ are the same and the proof is complete.

4. We shall prove the following theorem:

THEOREM 3. For every form $f$ representing primitively an integer a prime to $\Omega$ and any particular integer $a^{\prime} \equiv a(\bmod 16 H h \mu)$, where $h$ and $\mu$ are defined in Theorem 2, there exists a form of the same Hessian and genus representing primitively $a^{\prime}$.

The method of proof here is a kind of generalization of that used by Dirichlet, as mentioned in the introduction to this paper.

We may consider $f$ to be in the form of $f$ in Theorem 1 with $a_{1}$ replaced by $a$. Then $H=a \delta-b s^{2}$ where $\delta=b c-r^{2}=\Omega^{2} d t, b=\Omega m \beta$ (see Theorem 1), $d$ is a power of 2 and $t$ is odd. Take $t=t_{1} t_{2}$, where $t_{2}$ is the greatest factor of $t$ prime to $H$. For $a^{\prime}$ then we seek integers $b^{\prime}, c^{\prime}$ and $r^{\prime}$ satisfying the conditions of Theorem 2 and such that $H=a^{\prime} \delta^{\prime}-b^{\prime} s^{2}$ where $\delta^{\prime}=b^{\prime} c^{\prime}-r^{\prime 2}=\Omega^{2} d t^{\prime}, b^{\prime}=\Omega m b_{1}$, $b_{1}$ prime to $2 h a^{\prime}$, and $t^{\prime}$ is odd. Take $t^{\prime}=\tau+16 h H m t_{1} \Omega s^{2} k$, where $\tau$ is chosen so that $a^{\prime} \delta^{\prime} \equiv a \delta \quad\left(\bmod 16 H h \Omega^{2} t_{1} d \mu m s^{2}\right)$. This is possible since this congruence is implied by $a^{\prime} t^{\prime} / \mu \equiv a t / \mu \quad\left(\bmod 16 H h t_{1} m s^{2}\right)$ and $a^{\prime} / \mu$ as well as $a / \mu$ is prime to the modulus. (Any factor common to $s$ and $a$ divides $H$.) Note that this congruence implies that $t^{\prime} / t_{1}$ is an integer prime to $h$, that $t^{\prime} / t_{1} \equiv t / t_{1} \quad(\bmod$ $16 H h)$, and thus $\delta \equiv \delta^{\prime}\left(\bmod 16 H h \Omega^{2} d t_{1}\right)$.

Then $b_{1}=16 h H d t_{1} \Omega^{2} a^{\prime} k+\left(a^{\prime} \Omega^{2} d \tau-H\right) /\left(\Omega m s^{2}\right)$, since $H=a^{\prime} \delta^{\prime}-b^{\prime} s^{2}$. In view of the choice of $\tau, b_{1}$ is an integer and $b_{1} \equiv \beta(\bmod 16 h \Omega)$. Now the second member on the right of the last equation involving $b_{1}$ is an integer prime to $2 H$ since $\beta$ is, and is prime to $a^{\prime}$ since any factor common to $a^{\prime}$ and $b_{1}$ divides $H$. Thus the second member on the right and the coefficient of $k$ are relatively prime, and by a classical theorem in the theory of numbers, we may choose $k$ so that $b_{1}=p$, a prime not dividing $2 H$. Also $\beta$ is prime to $\delta$ since any factor common to $\beta$ and $\delta$ would divide $H$. For a similar reason $p$ is prime to $\delta^{\prime}$. Since $b c-r^{2}=\delta,(-\delta \mid \beta)=1$.

Now $\left(-\delta^{\prime} \mid p\right)=\left(-d t^{\prime} \mid p\right)=(-d \mid p)\left(p \mid t^{\prime}\right)(-1)^{\alpha}$, where $\alpha=\left(t^{\prime}-1\right)(p-1) / 4$ $\equiv(t-1)(\beta-1) / 4 \quad(\bmod 2)$. Take $H=H^{\prime} H^{\prime \prime}, m=m^{\prime} m^{\prime \prime}, \Omega=\Omega^{\prime} \Omega^{\prime \prime}$, the first factor in each equality being the greatest odd factor in the left member. Take $t_{3}^{\prime}=\left(t^{\prime}, H\right), t_{3}=(t, H), t^{\prime} / t_{3}^{\prime}=t_{4}^{\prime}, t / t_{3}=t_{4}$. Then $t_{1} \equiv 0 \quad\left(\bmod t_{3}^{\prime}\right) \equiv 0$ $\left(\bmod t_{3}\right)$ and $t^{\prime} / t_{3} \equiv t / t_{3}(\bmod 16 H h)$. Thus $t_{3}=t_{3}^{\prime}$ and $t_{4}^{\prime} \equiv t_{4} \quad(\bmod 8 H h)$. Let $t_{3}=t_{13} t_{23}$, where $t_{23}$ is the greatest odd factor of $t_{3}$. Now, noting that $\Omega m s^{2} / t_{3}^{\prime}$ is prime to $t_{4}$ and $t_{4}^{\prime}$ since $H / t_{3}^{\prime}$ is, and $H=a^{\prime} \Omega^{2} d t^{\prime}-\Omega m p s^{2}$, we have 


$$
\begin{aligned}
\left(-\delta^{\prime} \mid p\right)= & (-d \mid p)\left(\left\{-H^{\prime \prime} \Omega^{\prime \prime} m^{\prime \prime} / t_{13}^{2}\right\} \mid t_{4}^{\prime}\right)\left(\left\{H^{\prime} m^{\prime} \Omega^{\prime} / t_{23}{ }^{2}\right\} \mid t_{4}^{\prime}\right) \\
& \cdot\left(p \mid t_{3}^{\prime}\right)(-1)^{\alpha} \\
= & (-d \mid p)\left(\left\{-H^{\prime \prime} \Omega^{\prime \prime} m^{\prime \prime} / t_{13}{ }^{2}\right\} \mid t_{4}^{\prime}\right)\left(p \mid t_{3}^{\prime}\right)\left(t_{4}^{\prime} \mid\left\{H^{\prime} m^{\prime} \Omega^{\prime} / t_{23}{ }^{2}\right\}\right)(-1)^{\gamma}
\end{aligned}
$$

where

$$
\gamma=\alpha+\left(t_{4}^{\prime}-1\right)\left(H^{\prime} m^{\prime} \Omega^{\prime}-1\right) / 4 \equiv \alpha+\left(t_{4}-1\right)\left(H^{\prime} m^{\prime} \Omega^{\prime}-1\right) / 4 \quad(\bmod 2) .
$$

Therefore

$$
\begin{gathered}
\left(-\delta^{\prime} \mid p\right)=(-d \mid p)\left(\left\{-H^{\prime \prime} \Omega^{\prime \prime} m^{\prime \prime} / t_{13}{ }^{2}\right\} \mid t_{4}\right)\left(p \mid t_{3}\right)\left(t_{4} \mid\left\{H^{\prime} m^{\prime} \Omega^{\prime} / t_{23}{ }^{2}\right\}\right), \\
(-1)^{\gamma}=(-d \mid p)\left(\left\{-H^{\prime \prime} \Omega^{\prime \prime} m^{\prime \prime} / t_{13}{ }^{2}\right\} \mid t_{4}\right)\left(p \mid t_{3}\right)\left(\left\{H^{\prime} m^{\prime} \Omega^{\prime} / t_{23}{ }^{2}\right\} \mid t_{4}\right), \\
(-1)^{\alpha}=(-d \mid \beta)(\beta \mid t)(-1)^{\alpha},
\end{gathered}
$$

since $\left(p \mid t_{3}\right)=\left(\beta \mid t_{3}\right), t_{3}$ being a factor of $H$. Thus $\left(-\delta^{\prime} \mid p\right)=(-d \mid \beta)(t \mid \beta)$ $=(-\delta \mid \beta)=1$.

Therefore there exists a $\rho$ such that $-\delta^{\prime} \equiv \rho^{2}(\bmod p)$. Choose $r^{\prime} \equiv \rho$ $(\bmod p)$ and $\equiv r\left(\bmod 16 \Omega^{2} d h H\right)$. Then $\delta+r^{2} \equiv \delta^{\prime}+r^{\prime 2}(\bmod 16 H \Omega)$, showing that $\delta^{\prime}+r^{\prime 2} \equiv 0 \quad(\bmod \Omega m)$, and we have shown the existence of a $c^{\prime}$ such that $\delta^{\prime}=\Omega m p c^{\prime}-r^{\prime 2}$. Noting that $\delta=\Omega m \beta c-r^{2}$, we have $\Omega m p c^{\prime} \equiv \Omega m \beta c$ $\left(\bmod 16 H h \Omega^{2} d\right)$ and thus $p c^{\prime} \equiv \beta c \quad(\bmod 16 H h \Omega d / m)$. Noting that $2 H \equiv 0$ $(\bmod m)$ we have $c^{\prime} \equiv c \quad(\bmod 8 h \Omega)$ and the conditions of Theorem 2 that the forms $f$ and $f^{\prime}$ be of the same genus are satisfied. Since the leading coefficient of $f^{\prime}$ is $a^{\prime}$, the representation of $a^{\prime}$ by $f^{\prime}$ is primitive.

THEOREM 3a. For every form $f$ representing an integer a prime to $\Omega$ and any particular $a^{\prime} \equiv a \quad(\bmod 8 h \mu)$, there exists a form $f^{\prime}$ of the same genus and Hessian representing $a^{\prime}$.

$16 H h \mu$ may be replaced by $8 h \mu$ in the statement of Theorem 3 since $f \equiv a(\bmod 16 H h \mu)$ solvable implies $f \equiv a(\bmod 8 h \mu)$ solvable and, by Lemma 17 of the previous paper* $f \equiv a(\bmod 8 h \mu)$ solvable implies $f \equiv a$ $(\bmod 16 H h \mu)$ solvable.

Suppose $a=k^{2} a_{1}$, where $f$ represents $a_{1}$ primitively and $k=k_{1} k_{2}, k_{2}$ being the largest factor of $k$ prime to $2 h$. Then $a_{1} k_{1}^{2} / \mu$ is prime to $2 h$ and $f \equiv a_{1} k_{2}{ }^{2}$ $\left(\bmod 8 h \mu / k_{1}^{2}\right)$ is solvable.

Now since the quadratic characters of $a_{1} k_{1}^{2} / \mu$ and of $a_{1} k_{1}{ }^{2} k_{2}^{2} / \mu$ with respect to the factors of $h$ are the same and since they are congruent $\bmod 8$, we have, by the lemmas of the previous paper, that $f \equiv a_{1} \cdot\left(\bmod 8 h \mu / k_{1}^{2}\right)$ is solvable. Then $f \equiv a_{1} k_{2}{ }^{2} \quad\left(\bmod 8 h \mu / k_{1}{ }^{2}\right)$ is solvable primitively, for, since $f$ represents $a_{1}$ primitively, we may transform $f$ to a form $f_{1}$ having its leading coefficient $a_{1}$ and a primitive solution of $f_{1} \equiv a_{1} k_{2}{ }^{2} \quad\left(\bmod 8 h \mu / k_{1}^{2}\right)$ is $x=k_{2}$,

* See the sixth footnote of the Introduction. 
$y=z=8 h \mu / k_{1}{ }^{2}$. Thus, from Theorem 3, there exists a form $f^{\prime}$ of the same genus representing $a^{\prime} / k_{1}^{2} \equiv a_{1} k_{2}^{2} \quad\left(\bmod 8 h \mu / k_{1}^{2}\right)$. Thus $f^{\prime}$ represents $a^{\prime}$.

5. We need two further lemmas.

Lemma 5. Any form $f$ with $\Omega=p \Omega^{\prime} \not \equiv 0\left(\bmod p^{2}\right)$ and $H \not \equiv 0\left(\bmod p^{3}\right)$, i.e., $H / \Omega^{2}=\Delta \neq 0 \quad(\bmod p)$, is equivalent to a form $f_{1} \equiv \alpha x^{2}+b^{\prime} p y^{2}+c^{\prime} p z^{2}$ $+2 r^{\prime} p y z\left(\bmod p^{3}\right)$ where $\left(b^{\prime} c^{\prime} \mid p\right)=-1$ and $\alpha \neq 0(\bmod p)(p$ is an odd prime).

In $f=\left(\alpha, b_{1}, c_{1}, 2 r_{1}, 2 s_{1}, 2 t_{1}\right)$ we may consider $\alpha$ to be prime to $p$. Replace $x$ by $x+\tau y+\sigma z$, choosing $\tau$ and $\sigma$ so the coefficients of $2 x y$ and $2 x z$ are $\equiv 0$ $\left(\bmod p^{3}\right)$, and have $f \equiv \alpha x^{2}+b y^{2}+c z^{2}+2 r z y \quad\left(\bmod p^{3}\right)$. Now $\Omega \equiv 0 \quad(\bmod p)$ implies $b=p b^{\prime}, c=p c^{\prime}, r=p r^{\prime}$ and $f \equiv \alpha x^{2}+b^{\prime} p y^{2}+c^{\prime} p z^{2}+2 r^{\prime} p y z \quad\left(\bmod p^{3}\right)$. If $\left(b^{\prime} c^{\prime} \mid p\right)=-1$ the lemma is proved.

If $b^{\prime} \equiv c^{\prime} \equiv 0 \quad(\bmod p)$ we know $r^{\prime} \neq 0 \quad(\bmod p)$, since $\Omega \neq 0 \quad\left(\bmod p^{2}\right)$ and replacement of $z$ by $y+z$ in $b^{\prime} y^{2}+c^{\prime} z^{2}+2 r^{\prime} y z$ yields a form with the coefficient of $y^{2}$ prime to $p$. This therefore reduces to the case below.

If $b^{\prime}$ or $c^{\prime}$ is prime to $p$, interchange $y$ and $z$ if necessary to have $b^{\prime}$, the coefficient of $y^{2}$, prime to $p$. In $f$ replace $y$ by $y+\tau z$ and see that the coefficients of $y^{2}, x y, x z$ remain unaltered $\bmod p^{3}$ and that of $p z^{2}$ becomes $b^{\prime} \tau^{2}+2 r^{\prime} \tau+c^{\prime}$. To show that we may choose $\tau$ so that $b^{\prime}\left(b^{\prime} \tau^{2}+2 r^{\prime} \tau+c^{\prime}\right)=\left(b^{\prime} \tau+r^{\prime}\right)^{2}+b^{\prime} c^{\prime}-r^{\prime 2}$ is a non-residue of $p$, it is only necessary to show that for any $a$ prime to $p$ there exists an $x$ such that $x^{2}+a$ is a non-residue of $p$. $\left(b^{\prime} c^{\prime}-r^{\prime 2}\right.$ is prime to $p$ since $H \equiv p^{2} \alpha\left(b^{\prime} c^{\prime}-r^{\prime 2}\right) \not \equiv 0\left(\bmod p^{3}\right)$.) This is obvious if $(a \mid p)=-1$. If $(a \mid p)=1$ the values $0,1, \cdots,(p-1) / 2$ of $x$ give $(p+1) / 2$ incongruent values of $x^{2}+a(\bmod p)$, one of which must be a non-residue, unless, for one of these values of $x, x^{2}+a \equiv 0 \quad(\bmod p)$, and for the other values, $x^{2}+a$ ranges in value over all the residues of $p$. This can happen only if $(-a \mid p)=1$, i.e. if $p \equiv 1(\bmod 4)$, and if, for every residue $R$ of $p, x^{2}+a \equiv-R a(\bmod p)$ is solvable for $x,-R a$ being a residue of $p$. This is true only if $x^{2} \equiv-a(R+1)$ $(\bmod p)$ is solvable for every $R$, i.e. if, for every $R \not \equiv-1(\bmod p), R+1$ is a residue of $p$. If this were true, since 1 is a residue of $p$, every positive integer less than $p$ would be a residue of $p$ which is false.

LeMma 6. If for a form $f_{1}=\left(\alpha^{\prime}, b, c, 2 r, 2 s, 2 t\right), \alpha^{\prime}$ odd,$\Omega_{1} \equiv 2 \quad(\bmod 4)$ and $\Delta_{1} \equiv 2,4$ or $6 \quad(\bmod 8)$, it is true that $f_{1} \sim f_{1}^{\prime} \equiv \alpha^{\prime} x^{2}+2 \beta^{\prime} y^{2}+2 \Delta_{1} \gamma^{\prime} z^{2}(\bmod$ $\left.128 \Delta_{1} \Omega_{1}\right)$ and $f_{1} \sim f_{1}^{\prime \prime} \equiv \alpha^{\prime} x^{2}+2 \beta^{\prime \prime} y^{2}+2 \Delta_{1} \gamma^{\prime \prime} z^{2} \quad\left(\bmod 128 \Delta_{1} \Omega_{1}\right)$ where $\beta^{\prime} \beta^{\prime \prime} \equiv 1$ $+\Delta_{1} \beta^{\prime} \gamma^{\prime}(\bmod 8)$ and $\alpha^{\prime}$ prime to $2 H$.

Since $\Omega_{1} \equiv \Delta_{1} \equiv 0 \quad(\bmod 2)$ implies that $f_{1}$ and $F_{1}$ are properly primitive, $f_{1} \sim f_{1}^{\prime}$ above from Lemma 12 in the previous paper, and $\beta^{\prime}=\Omega_{1} \beta / 2, \gamma^{\prime}=\gamma \Omega_{1} / 2$, where $\alpha^{\prime} \beta \gamma$ is prime to $2 \Omega_{1} \Delta_{1}$. Thus $\beta^{\prime} \gamma^{\prime}$ is odd. The substitution $y=y^{\prime}$, $z=y^{\prime}+z^{\prime}$ transforms $\beta^{\prime} y^{2}+\Delta_{1} \gamma^{\prime} z^{2}$ into $\left(\beta^{\prime}+\Delta_{1} \gamma^{\prime}\right) y^{\prime 2}+c z^{\prime 2}+2 r y^{\prime} z^{\prime}$. Let $\beta^{\prime \prime}=\beta^{\prime}$ 
$+\Delta_{1} \gamma^{\prime} \equiv 1(\bmod 2)$ and replace $y^{\prime}$ by $y^{\prime}+\tau z^{\prime}$, choosing $\tau$ so that the coefficient of $2 y^{\prime} z^{\prime}$ is $\equiv 0 \quad\left(\bmod 64 \Delta_{1} \Omega_{1}\right)$. This does not alter the coefficient of $y^{\prime 2}$ and gives $f_{1}^{\prime} \sim f_{1}^{\prime \prime} \equiv \alpha^{\prime} x^{2}+2 \beta^{\prime \prime} y^{2}+2 c^{\prime} z^{2} \quad\left(\bmod 128 \Delta_{1} \Omega_{1}\right)$, where $\beta^{\prime} \beta^{\prime \prime} \equiv 1$ $+\Delta_{1} \beta^{\prime} \gamma^{\prime}(\bmod 8)$. Now $H \equiv 4 \alpha^{\prime} \beta^{\prime \prime} c^{\prime}\left(\bmod 128 \Delta_{1} \Omega_{1}\right)$, i.e., $H /\left(4 \Omega^{\prime}\right) \equiv \alpha^{\prime} \beta^{\prime \prime} c^{\prime} / \Omega^{\prime}$ $\left(\bmod 64 \Delta_{1}\right)$ where $\Omega^{\prime}=\Omega_{1} / 2$. We have $\alpha^{\prime} \beta^{\prime \prime} / \Omega^{\prime} \neq 0\left(\bmod \Delta_{1}\right)$ and thus $c^{\prime} \equiv H /\left(4 \Omega^{\prime}\right) \equiv 0 \quad\left(\bmod \Delta_{1}\right)$ and the lemma is proved.

6. We use the following abbreviation throughout the remainder of the paper: $g=f / n$ means "the multiples of $n$ represented by $f$ are $n$ multiplied by the integers represented by $g . "$

THEOREM 4. For any genus $G$ of primitive forms $f$ and Hessian $H$ and any prime factor $q$ of $\Omega$ there exists a genus $G_{1}\left(\right.$ or $\left.G_{2}\right)$ of primitive forms $f_{1}\left(\right.$ or $\left.f_{2}\right)$ of Hessian $H / q$ (or $\left.H / q^{4}\right)$ such that the multiples of $q$ (or $\left.q^{2}\right)$ represented by a form $f$ of $G$ are $q\left(\right.$ or $\left.q^{2}\right)$ multiplied by the integers represented by a form $f_{1}\left(\right.$ or $\left.f_{2}\right)$ of $G_{1}\left(\right.$ or $\left.G_{2}\right)$.

Use the part of the theorem in parentheses if $\Omega \equiv 0 \quad\left(\bmod q^{2}\right)$. Furthermore if $\Omega \neq 0 \quad\left(\bmod q^{2}\right), \Omega_{1}$, the $\Omega$-factor of $f_{1}$, is $\Omega$ or $\Omega / q$ according as $\Delta \equiv 0$ $(\bmod q)$ or not, i.e. according as $H \equiv 0 \quad\left(\bmod q^{3}\right)$ or not. If $\Omega \equiv 0 \quad\left(\bmod q^{2}\right)$, $\Omega_{2}=\Omega / q^{2}$. Note that by the notation explained above $f_{1}=f / q$ and $f_{2}=f / q^{2}$.

I. $q=p$ an odd prime. From Lemma 12 of the previous paper we may consider $f \equiv \alpha x^{2}+\beta \Omega y^{2}+\gamma \Omega \Delta z^{2} \quad\left(\bmod p^{t+1}\right)$ where $t$ is the highest power of $p$ in $H$ and $\alpha \beta \gamma \equiv 1 \quad\left(\bmod p^{t+1}\right)$. Then $f \equiv 0 \quad(\bmod p)$ implies $x=p x_{1}$ and, making the substitution in $f$ and dividing by $p$, we have $f_{1} \equiv p \alpha x_{1}{ }^{2}+\beta \Omega y^{2} / p$ $+\gamma \Omega \Delta z^{2} / p\left(\bmod p^{i}\right) . f_{1}=f / p$.

First if $\Omega \neq 0 \quad\left(\bmod p^{2}\right), f_{1}$ is primitive since the coefficients are altered only by multiples of $p$ or $1 / p$ and $\Omega / p \neq 0 \quad(\bmod p)$. The Hessian of $f_{1}$ is $H / p$. Take the genus $G_{1}$ to be the genus of $f_{1}$. Now the progressions (1) of the previous paper associated with $f_{1}$ are those obtained by dividing by $p$ all the multiples of $p$ in the progressions (1) associated with $f$. By the theorem of the previous paper all forms $f$ of genus $G$ have the same progressions (1) associated with them. Thus all forms $f_{1}$ obtained by the above process from forms $f$ of $G$ have the same progressions (1) associated with them, and, by the same theorem, are thus of the same genus $G_{1}$. Conversely consider any form $f_{1}^{\prime}$ of genus $G_{1}$ and Hessian $H / p$. Below we prove the existence of a form $f^{\prime}$ for which $f^{\prime} / p=f_{1}^{\prime}$ and the quadratic character of the integers prime to $p$ represented by $f^{\prime}$ is the same as that of the integers prime to $p$ represented by $f$. This proves that the progressions (1) associated with $f^{\prime}$ and $f$ are the same and therefore that $f$ and $f^{\prime}$ are of the same genus.

Take $f \equiv \alpha x^{2}+\beta \Omega y^{2}+\gamma \Omega \Delta z^{2} \quad\left(\bmod p^{t+1}\right)$ to be a form of genus $G$.

A. $\Omega_{1}=\Omega=p \Omega^{\prime}$ and $\Delta_{1}=\Delta / p \equiv 0(\bmod p)$. Then by Lemma 12 of the 
previous paper, we take $f_{1}^{\prime} \equiv \alpha^{\prime} x^{2}+\beta^{\prime} \Omega y^{2}+\gamma^{\prime} \Omega \Delta_{1} z^{2} \quad\left(\bmod p^{t}\right)$. Multiply $f_{1}^{\prime}$ by $p$, replace $p y$ by $y$ and have $f^{\prime} \equiv \alpha^{\prime} p x^{2}+\beta^{\prime} \Omega^{\prime} y^{2}+\gamma^{\prime} \Omega \Delta z^{2} \quad\left(\bmod p^{t+1}\right)$ and $f^{\prime} / p=f_{1}^{\prime}$. Furthermore $\left(\beta^{\prime} \Omega^{\prime} \mid p\right)=(\alpha \mid p)$, for the multiples of $p$ represented by $f_{1}^{\prime}$ and by $f_{1}$ must be of the same character since $f_{1}^{\prime}$ and $f_{1}$ are of the same genus and $f_{1}^{\prime} / p \equiv \alpha^{\prime} p x_{1}{ }^{2}+\beta^{\prime} \Omega^{\prime} y^{2}+\gamma \Omega^{\prime} \Delta_{1} z^{2}\left(\bmod p^{t-1}\right)$, while $f_{1} / p \equiv \alpha x_{1}{ }^{2}$ $+\beta \Omega y_{1}{ }^{2}+\gamma \Omega \Delta z^{2} / p^{2}\left(\bmod p^{t-1}\right)$. Thus the conditions on $f^{\prime}$ required above are satisfied.

B. $\Omega_{1}=\Omega=p \Omega^{\prime}$ and $\Delta_{1}=\Delta / p \not \equiv 0 \quad(\bmod p)$. Then, by Lemma 5 , we may consider $f_{1}^{\prime} \equiv \alpha^{\prime} x^{2}+b^{\prime} p y^{2}+c^{\prime} p z^{2}+2 r^{\prime} p y z \quad\left(\bmod p^{3}\right)$ with $\left(b^{\prime} c^{\prime} \mid p\right)=-1$. Thus we can interchange $y$ and $z$ if necessary to make $\left(b^{\prime} \mid p\right)=(\alpha \mid p)$. Then multiply $f_{1}^{\prime}$ by $p$ and replace $p y$ by $y$, having $f^{\prime} \equiv \alpha^{\prime} p x^{2}+b^{\prime} y^{2}+c^{\prime} p^{2} z^{2}+2 r^{\prime} p^{2} y z \quad(\bmod$ $\left.p^{3}\right)$, where $f^{\prime} / p=f_{1}^{\prime}$ and, as above, is of genus $G$.

C. $\Omega_{1}=\Omega / p$. Then $\Delta_{1}=\Delta p \neq \equiv \quad\left(\bmod p^{2}\right)$ for $\Omega_{1} F_{1} \equiv \beta \gamma \Omega^{2} \Delta x^{2} / p^{2}+\alpha \gamma \Omega \Delta y^{2}$ $+\alpha \beta \Omega z^{2} \quad(\bmod p)$ and $\Delta \equiv 0 \quad(\bmod p)$ contradicts $\Omega_{1} \neq 0 \quad(\bmod p)$. Thus we take $f_{1}^{\prime} \equiv \alpha^{\prime} x^{2}+\beta^{\prime} \Omega_{1} y^{2}+\gamma^{\prime} \Omega \Delta z^{2} \quad\left(\bmod p^{3}\right)$. Multiply through by $p$, replace $p z$ by $z$, and have $f^{\prime} \equiv p \alpha^{\prime} x^{2}+\beta^{\prime} \Omega y^{2}+\gamma^{\prime} \Delta \Omega_{1} z^{2} \quad\left(\bmod p^{3}\right)$. Now $f^{\prime} / p=f_{1}^{\prime}$ and $\left(\gamma^{\prime} \Delta \Omega_{1} \mid p\right)=(\alpha \mid p)$ for $f_{1}^{\prime}$ and $f_{1}$, being of the same genus, have the same progressions (1) associated with them, and, by Lemmas 4 and 5 of the previous paper, $(-\beta \gamma \Delta \mid p)=\left(-\alpha^{\prime} \beta^{\prime} \Omega_{1} \mid p\right)$, i.e. $(\alpha \Delta \mid p)=\left(\gamma^{\prime} \Omega_{1} \mid p\right)$.

Second, if $\Omega \equiv 0 \quad\left(\bmod p^{2}\right), f_{1} / p=f_{2} \equiv \alpha x_{1}{ }^{2}+\beta \Omega y^{2} / p^{2}+\gamma \Delta \Omega z^{2} / p^{2} \quad(\bmod$ $\left.p^{t-1}\right)$, where the Hessian of $f_{2}$ is $H / p^{4}$ and $\Omega_{2}=\Omega / p^{2}$. Now $f / p^{2}=f_{2}$ and, as above, we define $G_{2}$ to be the genus of $f_{2}$. All forms $f_{2}$ so obtained from forms of genus $G$ are of the same genus $G_{2}$. Conversely any $f_{2}^{\prime}$ of genus $G_{2}$ may be taken $f_{2}^{\prime} \equiv \alpha^{\prime} x^{2}+\beta^{\prime} \Omega y^{2} / p^{2}+\gamma^{\prime} \Omega \Delta z^{2} / p^{2}\left(\bmod p^{t-1}\right)$ where $\left(\alpha^{\prime} \mid p\right)=(\alpha \mid p)$, for $f_{2}^{\prime}$ represents some integer $\alpha^{\prime}$ prime to $2 H$ for which $\left(\alpha^{\prime} \mid p\right)=(\alpha \mid p)$, thus represents some such integer primitively, and, by Lemma 1 of this paper, is equivalent to a form with leading coefficient $\alpha^{\prime}$. Smith's process (ibid., pp. 460-462) by which $f^{\prime}$ is reduced to the form above leaves such a leading coefficient unaltered as is pointed out in the corollary to Lemma 12 of the previous paper. Now multiply the above form of $f_{2}^{\prime}$ by $p$ and replace $p x$ by $x$ having $f^{\prime} \equiv \alpha^{\prime} x^{2}$ $+\beta^{\prime} \Omega y^{2}+\gamma^{\prime} \Omega z^{2}\left(\bmod p^{t+1}\right)$, where $f^{\prime} / p^{2}=f_{2}^{\prime} . f^{\prime}$ represents no multiple of $p \neq 00\left(\bmod p^{2}\right),\left(\alpha^{\prime} \mid p\right)=(\alpha \mid p)$, and therefore the progressions $(1)$ of the previous paper associated with $f^{\prime}$ and $f$ are the same and $f^{\prime}$ and $f$ are of the same genus.

II. $q=2$. Since $\Omega \equiv 0 \quad(\bmod 2)$ we know $f$ is properly primitive. If further $F$ is properly primitive we apply Lemma 12 of the previous paper as for $q=p$ and have $f \equiv \alpha x^{2}+\beta \Omega y^{2}+\gamma \Omega \Delta z^{2} \quad\left(\bmod 2^{3+t}\right), f_{1} \equiv 2 \alpha x_{1}{ }^{2}+\Omega \beta y^{2} / 2+\gamma \Delta z^{2} \Omega / 2$ $\left(\bmod 2^{2+t}\right)$ and $f / 2=f_{1} . F$ is properly primitive if $\Delta \equiv 0 \quad(\bmod 2)$.

First, if $\Omega \equiv 2 \quad(\bmod 4)$ and $F$ is properly primitive, 2 may be substituted for $p$ in the corresponding discussion for $q=p$ if "the quadratic character of 
an odd integer $\bmod 2 "$ we interpret to mean the congruence satisfied $\bmod 8$, i.e. $a_{1}$ and $a_{2}$ are of the "same quadratic character with respect to 2 " if $a_{1} \equiv a_{2}$ $(\bmod 8)$.

A. $\Omega_{1}=\Omega=2 \Omega^{\prime}$ and $\Delta_{1}=\Delta / 2 \equiv 0 \quad(\bmod 8)$. Using Lemma 12 of the previous paper we may take $f_{1}^{\prime} \equiv \alpha^{\prime} x^{2}+2 \beta^{\prime} \Omega^{\prime} y^{2}+2 \gamma^{\prime} \Omega^{\prime} \Delta_{1} z^{2} \quad\left(\bmod 8 \Delta_{1}\right)$. Multiply by 2 , replace $2 y$ by $y$, and have $f^{\prime} \equiv 2 \alpha^{\prime} x^{2}+\beta^{\prime} \Omega^{\prime} y^{2}+4 \gamma^{\prime} \Omega^{\prime} \Delta_{1} z^{2} \quad\left(\bmod 16 \Delta_{1}\right)$ and $f^{\prime} / 2=f_{1}^{\prime}$. Now the evens represented by $f_{1}^{\prime}$ and by $f_{1}$ satisfy the same congruences $\bmod 16$. Thus $f_{1}^{\prime} / 2 \equiv 2 \alpha^{\prime} x^{2}+\beta^{\prime} \Omega^{\prime} y^{2}+\gamma^{\prime} \Omega^{\prime} \Delta_{1} z^{2} \quad\left(\bmod 4 \Delta_{1}\right)$ and $f_{1} / 2$ $\equiv \alpha x_{1}{ }^{2}+2 \beta \Omega^{\prime} y^{2}+\gamma \Omega^{\prime} \Delta_{1} z^{2} \quad\left(\bmod 4 \Delta_{1}\right)$ implies that $\beta^{\prime} \Omega^{\prime}$ and $\beta^{\prime} \Omega^{\prime}+2 \alpha^{\prime}$ are congruent $(\bmod 8)$ in some order to $\alpha$ and $\alpha+2 \beta \Omega^{\prime}$, which proves that the odd integers represented by $f$ and $f^{\prime}$ satisfy the same congruences mod 8 .

B. $\Omega_{1}=\Omega=2 \Omega^{\prime}$ and $\Delta_{1}=\Delta / 2 \equiv 4(\bmod 8)$. Using Lemma 6 we may take $f_{1}^{\prime} \equiv \alpha^{\prime} x^{2}+2 \beta^{\prime} y^{2}+2 \Delta_{1} \gamma^{\prime} z^{2}\left(\bmod 128 \Delta_{1}\right)$ and also $f_{1}^{\prime} \sim f_{1}^{\prime \prime} \equiv \alpha^{\prime} x^{2}+2 \beta^{\prime \prime} y^{2}+2 \Delta_{1} \gamma^{\prime \prime} z^{2}$ $\left(\bmod 128 \Delta_{1}\right)$ where $\beta^{\prime} \beta^{\prime \prime} \equiv 5 \quad(\bmod 8)$. Now $f_{1}^{\prime}$ represents primitively an integer $\equiv \beta \Omega^{\prime} \quad(\bmod 8)$, since $f_{1}$ does. Then we may take $\alpha^{\prime} \equiv \beta \Omega^{\prime}(\bmod 8)$. Noting that $f_{1}^{\prime} \equiv \alpha^{\prime} x^{2}+2 \beta^{\prime} y^{2} \quad(\bmod 8)$ and $f_{1} \equiv 2 \alpha x_{1}{ }^{2}+\beta \Omega^{\prime} y^{2} \quad(\bmod 8)$ we see that $\alpha \equiv \beta^{\prime} \quad(\bmod 4)$. Replacing $f^{\prime}$ by $f^{\prime \prime}$ if necessary, we have $f_{1}^{\prime} \equiv \alpha^{\prime} x^{2}+2 b y^{2}$ $+2 \Delta_{1} \gamma^{\prime} z^{2}\left(\bmod 128 \Delta_{1}\right)$ with $b \equiv \dot{\alpha} \quad(\bmod 8)$ and $\alpha^{\prime} \equiv \beta \Omega^{\prime} \quad(\bmod 8)$. Multiplying $f_{1}^{\prime}$ by 2 and replacing $2 y$ by $y$ we have $f^{\prime} \equiv 2 \alpha^{\prime} x^{2}+b y^{2}+4 \Delta_{1} \gamma^{\prime} z^{2} \quad(\bmod$ $128 \Delta), f^{\prime} / 2=f_{1}^{\prime}$, and $f^{\prime}$ is of genus $G$.

C. $\Omega_{1}=\Omega=2 \Omega^{\prime}$ and $\Delta_{1}=\Delta / 2 \equiv 2(\bmod 4)$. Let $\Delta / 4=\Delta_{1} / 2=\Delta^{\prime}$. Here

$$
\begin{aligned}
\Delta^{\prime} f_{1}^{\prime} & \equiv \alpha^{\prime} x^{2}+2 \beta^{\prime} y^{2}+4 \gamma^{\prime} z^{2} \\
\Delta^{\prime} f & \equiv \alpha x^{2}+2 \beta y^{2}+8 \gamma z^{2}
\end{aligned}
$$
$(\bmod 128)$,

$\alpha^{\prime} \beta^{\prime} \gamma^{\prime} \equiv 1 \quad(\bmod 8)$ since $8 \alpha^{\prime} \beta^{\prime} \gamma^{\prime} \equiv \Delta^{\prime} \dot{H}_{1} \equiv 8 \quad(\bmod 64) . \quad$ Similarly $\alpha \beta \gamma \equiv 1$ $(\bmod 8)$. Now, since $f_{1}^{\prime} \equiv$ all odds $(\bmod 8)$ is solvable, we may consider $\alpha^{\prime} \equiv \beta$ $(\bmod 4)$. Since, by Lemma $6, \beta^{\prime}$ may be replaced by $\beta^{\prime \prime} \equiv 3 \beta^{\prime}(\bmod 4)$ and $\alpha^{\prime} \beta^{\prime \prime} \gamma^{\prime \prime} \equiv 1 \quad(\bmod 8)$, we have $\gamma^{\prime \prime} \equiv 3 \gamma^{\prime} \quad(\bmod 4)$. We therefore can make $\gamma^{\prime} \equiv \alpha^{\prime}$ or $3 \alpha^{\prime} \quad(\bmod 4)$ according as $\beta \equiv \gamma$ or $3 \gamma \quad(\bmod 4)$. Now the multiples of 4 represented by $\Delta^{\prime} f_{1}^{\prime}$ and by $\Delta^{\prime} f_{1}$ satisfy the same congruences (mod $32)$, i.e. the evens represented by $\Delta^{\prime} f_{1}^{\prime} / 2 \equiv 2 \alpha^{\prime} x^{2}+\beta^{\prime} y^{2}+2 \gamma^{\prime} z^{2}(\bmod 16)$ and by $\Delta^{\prime} f_{1} / 2 \equiv \alpha x^{2}+2 \beta y^{2}+2 \gamma z^{2} \quad(\bmod 16)$ satisfy the same congruences mod 16 . Thus, by the corollaries to Lemmas 10 and 11 of the previous paper, $\alpha^{\prime}+\gamma^{\prime} \equiv 2$ or $4(\bmod 8)$ if and only if $\beta+\gamma \equiv 2$ or $4(\bmod 8)$ in some order. If $\beta+\gamma \equiv 2$ $(\bmod 8)$, we see that $\beta \equiv \gamma \quad(\bmod 4)$, and, by the above choice of $\gamma^{\prime}, \alpha^{\prime}+\gamma^{\prime} \equiv 2$ $(\bmod 4)$ and therefore $\alpha^{\prime}+\gamma^{\prime} \equiv 2(\bmod 8)$. Similarly for all cases, the above choice of $\gamma^{\prime}$ is seen to make $\alpha^{\prime}+\gamma^{\prime} \equiv \beta+\gamma \quad(\bmod 8)$.

Now if $\beta+\gamma \equiv 2 a \quad(\bmod 8), \alpha \equiv 2 a \beta-1 \quad(\bmod 8)$ and the odds represented by $\Delta^{\prime} f$ are exclusively $\equiv 2 a \beta-1,2 \beta(a+1)-1 \quad(\bmod 8)$. Multiply $\Delta^{\prime} f_{1}^{\prime}$ by 2 , replace $2 y$ by $y$, and have $\Delta^{\prime} f^{\prime} \equiv 2 \alpha^{\prime} x^{2}+\beta^{\prime} y^{2}+8 \gamma^{\prime} z^{2} \quad(\bmod 128) . \Delta^{\prime} f^{\prime} / 2$ 
$=\Delta^{\prime} / f_{1}^{\prime}$, and the odds represented by $\Delta^{\prime} f^{\prime}$ are exclusively $\equiv \beta^{\prime}$ and $\beta^{\prime}+2 \alpha^{\prime}$ $(\bmod 8) . \quad \beta^{\prime} \equiv 2 a \alpha^{\prime}-1 \equiv 2 a \beta-1 \quad(\bmod 8)$ and $\beta^{\prime}+2 \alpha^{\prime} \equiv 2 \beta(a+1)-1 \quad(\bmod$ 8 ) show that the odds represented by $\Delta^{\prime} f^{\prime}$ and by $\Delta^{\prime} f$ satisfy the same congruences $(\bmod 8)$ and thus $f^{\prime}$ and $f$ are of the same genus.

D. $\Omega_{1}=\Omega=2 \Omega^{\prime}$ and $\Delta_{1}=\Delta / 2=\Delta^{\prime} \equiv 1 \quad(\bmod 2)$. Now $f_{1}$ represents $\beta \Omega^{\prime}$ and $\beta \Omega^{\prime}+2 \alpha \equiv 3 \beta \Omega^{\prime} \quad(\bmod 4)$ and thus from the form of $f_{1}$ in Lemma 14 of the previous paper we see this can happen only if $F_{1}$ is properly primitive. Furthermore for any form $f_{1}^{\prime}$ of genus $G_{1}, F_{1}^{\prime}$ is properly primitive since $f_{1}^{\prime}$, too, must represent integers $\equiv 1$ and $3(\bmod 4)$. Thus only $f_{1}^{\prime} \equiv \alpha^{\prime} x^{2}+2 \Omega^{\prime} \beta^{\prime} y^{2}$ $+2 \Omega^{\prime} \Delta^{\prime} \gamma^{\prime} z^{2}(\bmod 8)$ need be considered. Multiply by 2 , replace $2 y$ by $y$ and $f^{\prime} \equiv 2 \alpha^{\prime} x^{2}+\Omega^{\prime} \beta^{\prime} y^{2}+4 \Omega^{\prime} \Delta^{\prime} z^{2} \quad(\bmod 16) . f^{\prime}$ and $f \equiv 1,3,5$ or $7 \quad(\bmod 8)$ are solvable, $f^{\prime} / 2=f_{1}^{\prime}$, and thus $f^{\prime}$ and $f$ are of the same genus.

E. $\Omega_{1}=\Omega / 2 \equiv 1(\bmod 2)$ and $F$ properly primitive. Then $\Delta_{1}=2 \Delta \equiv 2$ (mod 4$)$ as in the case IC. Here $f_{1}$ represents an odd and thus the genus is composed of properly primitive forms. We have

$$
\begin{aligned}
\Delta f & =\Delta^{\prime} f \equiv \alpha x^{2}+2 \beta y^{2}+2 \gamma z^{2} & (\bmod 16) ; \\
\Delta f_{1} & \equiv 2 \alpha x_{1}^{2}+\beta y^{2}+\gamma z^{2}, \alpha \beta \gamma \equiv 1 & (\bmod 8) ; \\
\Delta f_{1}^{\prime} & \equiv \alpha^{\prime} x^{2}+\beta^{\prime} y^{2}+2 \gamma^{\prime} z^{2}, \alpha^{\prime} \beta^{\prime} \gamma^{\prime} \equiv 1 & (\bmod 8) .
\end{aligned}
$$

Since the odds represented by $\Delta f_{1}$ and $\Delta f_{1}^{\prime}$ satisfy the same congruences mod 8 , we have, from Lemmas 10 and 11 of the previous paper, $\beta+\gamma \equiv 2$ or 4 $(\bmod 8)$ if and only if $\alpha^{\prime}+\beta^{\prime} \equiv 2$ or $4(\bmod 8)$ in some order. Multiply $\Delta f_{1}^{\prime}$ by 2 , replace $2 z$ by $z$, and have $\Delta f^{\prime} \equiv 2 \alpha^{\prime} x^{2}+2 \beta^{\prime} y^{2}+\gamma^{\prime} z^{2} \quad(\bmod 16)$, and, by virtue of the corollaries to Lemmas 10 and 11 of the previous paper, the odds represented by $\Delta f^{\prime}$ and $\Delta f$ satisfy the same congruences $(\bmod 8) . \Delta f^{\prime} / 2=\Delta f_{1}^{\prime}$ and thus $f^{\prime}$ and $f$ are of the genus $G$.

F. $\Omega_{1}=\Omega / 2 \equiv 1(\bmod 2)$ and $F$ improperly primitive. Then, as above, $\Delta_{1}=2 \Delta \equiv 2(\bmod 4)$. Using Lemma 14 of the previous paper we take a form $f$ of genus $G$ as follows: $f \equiv a x^{2}+4 b y^{2}+4 c z^{2}+4 r y z \quad(\bmod 16)$ with $b r \equiv 1$ $(\bmod 2) . f / 2=f_{1} \equiv 2 a x_{1}{ }^{2}+2 b y^{2}+2 c z^{2}+2 r y z \quad(\bmod 8)$ and the genus $G_{1}$ of $f_{1}$ is composed of improperly primitive forms. Now from the proof of Lemma 13 of the previous paper we may take $f_{1}^{\prime} \equiv 2 \alpha x^{2}+2 b^{\prime \prime} x y+2 \alpha^{\prime} y^{2}+2 \beta z^{2} \quad(\bmod$ $16)$, where $\alpha b^{\prime \prime}$ is odd and $\beta=\gamma \Omega_{1} \Delta_{1} / 2 \equiv 1 \quad(\bmod 2)$ since $\gamma$ is an odd integer. Multiply $f_{1}^{\prime}$ by 2 , replace $2 z$ by $z$ and have $f^{\prime} \equiv \beta z^{2}+4 \alpha x^{2}+4 b^{\prime \prime} x y+4 \alpha^{\prime} y^{2}$ $(\bmod 16), f^{\prime} / 2=f_{1}^{\prime}$. Since $f^{\prime}$ represents only integers $\equiv \beta$ and $\beta+4 \alpha \quad(\bmod$ $8)$ and multiples of 4 , it remains to prove $\beta \equiv a(\bmod 4)$. This is done as follows: From the form of $f^{\prime}, H \equiv 4 \beta\left(4 \alpha \alpha^{\prime}-b^{\prime \prime 2}\right) \equiv 12 \beta \quad(\bmod 16)$, and likewise for $f, H \equiv 4 a\left(4 b c-r^{2}\right) \equiv 12 a \quad(\bmod 16) . \quad$ Thus $a \equiv \beta \quad(\bmod 4)$.

Second: $\Omega \equiv 0 \quad(\bmod 4)$. Then by Lemma 14 of the previous paper we may consider any form $f$ of genus $G$ to be of the form 


$$
f \equiv a x^{2}+2^{t_{1}} b y^{2}+2^{t_{1}} c z^{2}+2^{t_{1}+1} r y z
$$

where $\Omega \equiv 2^{t_{1}} \quad\left(\bmod 2^{t_{1}+1}\right), b$ is odd if $F$ is properly primitive and $b \equiv c \equiv r+1$ $\equiv 0(\bmod 2)$ if $F$ is improperly primitive. Now $t_{1} \geqq 2$ and $f \equiv 0(\bmod 2)$ implies $x=2 x_{1}$ and $f_{2}=f / 4 \equiv a x_{1}{ }^{2}+2^{t_{1}-2} b y^{2}+2^{t_{1}-2} c z^{2}+2^{t_{1}-1} r y z\left(\bmod 2^{t_{1}+1}\right)$. $H_{2}=H / 16, \Omega_{2}=\Omega / 4$ and $\Delta_{2}=\Delta$. Now $F_{2}$ is improperly primitive if and only if $b \equiv c \equiv r+1 \equiv 0 \quad(\bmod 2)$, i.e., if and only if $F$ is improperly primitive. Define the genus $G_{2}$ to be the genus of $f_{2}$. Take any form $f_{2}^{\prime}$ of genus $G_{2}$ and see by Lemma 14 of the previous paper that we may take

$$
f_{2} \equiv a^{\prime} x^{2}+2^{t_{1}-2} b^{\prime} y^{2}+2^{t_{1}-2} c^{\prime} z^{2}+2^{t_{1}-1} r^{\prime} y z
$$

$\left(\bmod 2^{t_{1}+1}\right)$,

where $a^{\prime} \equiv a \quad(\bmod 8), b^{\prime}$ is odd if $F_{2}$, and therefore $F$, is properly primitive, and $b^{\prime} \equiv c^{\prime} \equiv r^{\prime}+1 \equiv 0 \quad(\bmod 2)$ if $F_{2}$, and therefore $F$, is improperly primitive. Multiply by 4 , replace $2 x$ by $x$, and have

$$
f^{\prime} \equiv a^{\prime} x^{2}+2{ }^{t_{1}} b^{\prime} y^{2}+2{ }^{t_{1}} c^{\prime} z^{2}+2^{t_{1}+1} r^{\prime} y z
$$

$f^{\prime} / 4=f_{2}^{\prime}, f^{\prime}$ represents no $4 n+2$, and is of the same genus as $f$.

7. We prove the following theorem:

THEOREM 5. The integers represented by no primitive form $f$ of a given genus $G$ are exclusively those occurring in progressions (1), of the previous paper, associated with $f$.

Consider any integer $a$ included in none of the progressions (1) associated with $f$. It follows from the theorem of the previous paper that $f \equiv a(\bmod$ $8 h \mu)$ is solvable. If $a$ is prime to $\Omega$, Theorem $3 \mathrm{a}$ applies to prove the theorem above. Otherwise we proceed as follows.

First let $q$ be a prime factor of $a$ which, when squared, divides $\Omega$. Then $a \equiv 0 \quad\left(\bmod q^{2}\right)$ since reference to the proofs of the previous paper shows $\Omega \equiv 0 \quad\left(\bmod q^{2}\right)$ implies that $q a$ occurs in progressions $(1)$. Then there exists by Theorem 4 a form $f_{1}$ with $\Omega_{1}=\Omega / q^{2}$ and $H_{1}=H / q^{4}$ such that $f_{1}=f / q^{2}$ and thus $f_{1} \equiv a / q^{2} \quad\left(\bmod 8 h \mu / q^{2}\right)$. If $a / q^{2} \equiv 0 \quad(\bmod q)$ and $\Omega_{1} \equiv 0 \quad\left(\bmod q^{2}\right)$, we have $a / q^{2} \equiv 0 \quad\left(\bmod q^{2}\right)$ and the process may be repeated until, after $r$ times, $f_{r} \equiv a / q^{2 r} \quad\left(\bmod 8 h \mu / q^{2 r}\right)$ where $\Omega_{r}=\Omega / q^{2 r}, f_{r}=f / q^{2 r}, H_{r}=H / q^{4 r}$ and either $a / q^{2 r} \neq 00 \quad(\bmod q)$ or $\Omega_{r} \neq 0 \quad\left(\bmod q^{2}\right)$. If there is another prime factor, $q_{1}$, of $a$ which, when squared, divides $\Omega$, it is true that $\Omega_{r} \equiv 0 \quad\left(\bmod q_{1}{ }^{2}\right)$ and the above process may be applied to $f_{r}$. So this may be continued until we have the case below.

$g \equiv a / \mu_{1}^{2}=a^{\prime} \quad\left(\bmod 8 h \mu / \mu_{1}^{2}\right)$ where $\Omega_{g}=\Omega / \mu_{1}{ }^{2}, H_{o}=H / \mu_{1}^{4}, g=f / \mu_{1}{ }^{2}$ and no prime factor of $a^{\prime}$ is, when squared, a factor of $\Omega_{g}$. Let $q^{\prime}$ be a prime factor of $a^{\prime}$ dividing $\Omega_{g}$. Since $\Omega_{o} \neq 0 \quad\left(\bmod q^{\prime 2}\right)$, there exists a form $g_{1}=g / q^{\prime}$ of $\Omega_{g 1}=\Omega_{g}$ or $\Omega_{g} / q^{\prime}$ according as $H_{o} \equiv 0\left(\bmod q^{\prime 3}\right)$ or not. Then $g_{1} \equiv a^{\prime} / q^{\prime}$ 
$\left(\bmod 8 h \mu /\left\{\mu_{1}{ }^{2} q^{\prime}\right\}\right)$ is solvable. If $H_{\theta} \equiv 0 \quad\left(\bmod q^{\prime 3}\right)$ and $a^{\prime} / q^{\prime} \equiv \Omega_{g 1} \equiv 0 \quad(\bmod$ $q^{\prime}$ ), we repeat the process until after $t$ times we have $g_{t}=g / q^{\prime}$ where $\Omega_{o t}$ $=\Omega_{o} / q^{\prime t}$ or $\Omega_{o} /\left(q^{\prime}\right)^{t-1}, g_{t} \equiv a^{\prime} / q^{\prime t}\left(\bmod 8 h \mu /\left\{\mu_{1}^{2} q^{\prime t}\right\}\right)$ is solvable and either $a^{\prime} / q^{\prime t}$ or $\Omega_{g t}$ is prime to $q^{\prime}$. Then any other factor $q_{1}^{\prime}$ dividing $a^{\prime}$ and $\Omega_{o}$ divides $\Omega_{g t}$ and the above process may be applied to $g_{t}$. This may be carried through for every factor $q^{\prime}$ dividing $a^{\prime}$ and $\Omega_{g}$ until we have a form $g^{\prime}=f /\left\{\mu_{1}{ }^{2} \mu_{2}\right\}$ where $g^{\prime} \equiv a / \mu_{1}^{2} \mu_{2} \quad\left(\bmod 8 h \mu /\left\{\mu_{1}^{2} \mu_{2}\right\}\right), \Omega_{o}{ }^{\prime}=\Omega /\left(\mu_{1}{ }^{2} \mu_{2}^{\prime}\right)$, where $\mu_{2} \equiv 0 \quad(\bmod$ $\left.\mu_{2}^{\prime}\right), H_{g^{\prime}}=H /\left(\mu_{1}^{4} \mu_{2}\right)$ and $a /\left(\mu_{1}^{2} \mu_{2}\right)$ is prime to $\Omega_{g^{\prime}}$.

Then by Theorem 3a there exists a form $g^{\prime \prime}$ of the same genus and Hessian as $g^{\prime}$ which represents $a /\left(\mu_{1}{ }^{2} \mu_{2}\right)$. Following through the above process in the reverse order, applying the theorem of the previous paper at every step, we have the existence of a form $f^{\prime}$ of the same genus and Hessian as $f$ such that $f^{\prime} /\left(\mu_{1}{ }^{2} \mu_{2}\right)=g^{\prime \prime}$, which proves that $f^{\prime}$ represents $a$.

Corollary. A form $f$ of genus $G$ is regular if and only if $f$ represents all the integers represented by every form of $G$.

For suppose some integer $k$ is not represented by $f$ but is represented by some form $f^{\prime}$ of $G$. Then $k$ occurs in no progression (1) and thus $f \equiv k \quad(\bmod$ $N$ ) is solvable for $N$ arbitrary, every arithmetic progression containing $k$ contains an integer represented by $f$, and therefore, from the definition of regularity, $f$ is irregular.

Note. Whenever $f$ is the only reduced form of a genus it is regular, but, though most regular forms represent the only class in the genus, this is not always the case. See the following.

8. This section gives three examples.

I. $f=x^{2}+8 y^{2}+24 z^{2}$ and $g=x^{2}+2(2 y+x)^{2}+6(2 z+x)^{2}=8 y^{2}+9 x^{2}+24 z^{2}$ $+8 x y+24 x z$ are of the same genus. They are not of the same class since $g$ does not represent 1 . Every odd integer $n$ represented by $g$ is represented by $f$, for if $\left(x_{1}, y_{1}, z_{1}\right)$ is a solution of $g=n$, the \pm sign may be so chosen in $2 y_{1}+x_{1} \pm\left(2 z_{1}+x_{1}\right)=4 Y$ that the equation is solvable for $Y$ and $n=x_{1}{ }^{2}$ $+8\left(2 z+x_{1} \mp Y\right)^{2}+24 Y^{2}$. The evens represented by $f$ and $g$ are obviously the same. $f$ is regular since $f$ and $g$ represent the only classes in the genus, but $g$ is irregular.

II. The forms $x^{2}+3 y^{2}+6 z^{2}$ and $2 x^{2}+3 y^{2}+3 z^{2}$ are regular, for each represents the only class in the genus. (See the examples of the previous paper.)

III. The forms $f=(1,1,18)$ and $g=(2,2,5,0,-2,0)$ are of the same genus and both are irregular, for $f$ but not $g$ represents 1 and $g$ but not $f$ represents 7 .

Cornell University, ITHACA, N. Y. 